Editorial

\section{Special Issue on Pancreatic Imaging}

\author{
Raju Sharma ${ }^{1}$ Rajesh Arumugam² \\ ${ }^{1}$ Department of Radiology, All India Institute of Medical Sciences, \\ New Delhi, India \\ 2University of Leicester, College of Life Sciences, University \\ Hospitals of Leicester NHS Trust, Leicester, United Kingdom
}

J Gastrointestinal Abdominal Radiol ISGAR 2020;3:1

It is indeed a pleasure to have jointly edited this special issue of JGAR on pancreatic imaging. Pancreatic diseases, both inflammatory and neoplastic, are a significant cause of morbidity and mortality. There is a wide spectrum of inflammatory disorders, ranging from acute to chronic pancreatitis and including the more recently recognized autoimmune pancreatitis. Neoplastic pathology ranges from the ominous pancreatic cancer to cystic and neuroendocrine tumors. Cross-sectional imaging has become a critical tool in the workup of patients afflicted with pancreatic diseases. Rapid advances in imaging techniques have not only improved the diagnostic accuracy but have also enhanced our understanding of pathophysiology. CT plays a vital role in the diagnosis of acute pancreatitis, characterizes the related fluid collections and vascular complications like pseudoaneurysms, and has a major bearing on the treatment algorithms. The interventional radiologist today is at the forefront of treating these dreaded complications. Although imaging has improved the diagnosis and staging of pancreatic cancer, it has not made a significant dent in the overall outcome of this very aggressive tumor. Some problems continue to be elusive, including detecting carcinoma in the background of chronic pancreatitis and differentiating malignancy from mass-forming chronic pancreatitis.
This issue highlights the recent advances in the field of pancreatic MRI and endoscopic ultrasound (EUS). All the important disease entities have been dealt with by international and national experts in this field. The authors have distilled vital points from the literature and embellished it with their own

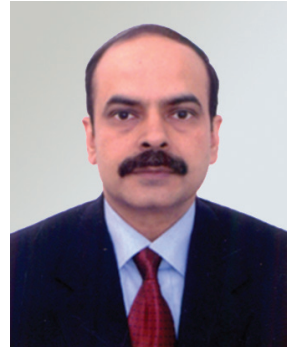

Raju Sharma

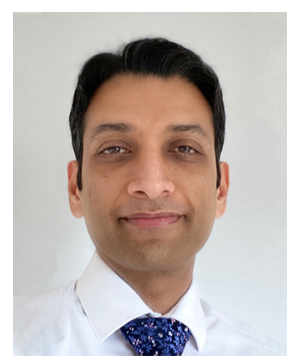

Rajesh Arumugam invaluable experience to provide a comprehensive overview to the reader. In addition to the common disease entities, there are articles devoted to the postoperative pancreas, pancreatic trauma, and rare tumors involving the pancreas. The role of interventional radiology in pancreatitis has also been highlighted.

To all the contributing authors, we would like to convey our sincere gratitude for having shared their experience with the readers! We value the insight offered by our clinical colleagues in the articles, as a multidisciplinary approach is crucial to any radiological practice. We hope the issue will provide an overview of the state-of-the-art imaging of this challenging yet fascinating organ. Happy reading!
(C)2020 Indian Society of Gastrointestinal and Abdominal Radiology

\section{License terms}

() $\odot \circledast$
Department of Radiology, All India

Institute of Medical Sciences,

New Delhi 110029, India

(e-mail: raju152@yahoo.com).
Raju Sharma, MD, MAMS, FICR,
DOI https://doi.org/

ISSN 2581-9933. 